\title{
Minimally Invasive Esophagectomy/Gastroesophagectomy for Cancer. Is it Safe in Nepalese Context?
}

\author{
Binay Thakur*, Li Hui, Mukti Devkota, Chen Xin and Robin Lama \\ Department of Surgical Oncology, BP Koirala Memorial Cancer Hospital, Bharatpur, Chitwan, Nepal
}

\begin{abstract}
Background: Minimally invasive approach for cancer of esophagus and gastroesophageal junction (GEJ) is gaining more popularity in the developed world mainly because of its better cosmetic results, lesser pain and lesser postoperative stay without compromising the radicality of the cancer surgery and survival. The aim of this study is to review the early outcome of this approach at BP Koirala Memorial Cancer Hospital.

Methods: Resectable tumors of GEJ and esophagus were treated primarily with surgery. Locally advanced tumors were considered for multimodality approach. Three ports were used for Video-assisted thoracoscopic (VATS) esophageal mobilization. Five ports were used for laparoscopic mobilization of stomach. Depending upon the feasibility, either a totally minimally invasive approach or a combination of minimally invasive approach with open technique was used. A $5 \mathrm{~cm}$ minilaparotomy was performed to retrieve the specimen.

Results: 34 patients with mean age of 57 years were reviewed. $9 \%, 38 \%, 29.5 \%$ and $23.5 \%$ of patients had malignancies of upper esophagus, middle esophagus, GEJ - I (distal esophagus) and GEJ - II (cardia), respectively. Primary surgery was performed in $91 \%$ of cases, whereas $9 \%$ underwent preoperative chemoradiation followed by surgery. VATS-laparotomy-neck (3-incision), thoracotomy-laparoscopy-neck (3-incision), laparoscopy-thoracotomy (2-incision), laparoscopic transhiatal-neck (2-incision), VATS-laparoscopy-neck (3-incision) and laparoscopyassisted (1-incision) approaches were used in $15 \%, 56 \%, 3 \%, 12 \%, 12 \%$ and $3 \%$, respectively. Mean number of dissected nodes was 22 and mean number of positive nodes was 6 . R0 resection was achieved in $94 \%$ of cases. The major postoperative complications were in-hospital mortality $(6 \%)$, anastomotic leak $(12 \%)$ and recurrent laryngeal nerve injury (6\%). The early (6 months) survival is $97 \%$.
\end{abstract}

Conclusion: Our results show, minimally invasive surgery is feasible, safe and the early outcome is promising though a longer follow-up is required for its strong recommendation in Nepalese context.

Keywords: Esophageal cancer; Laparoscopy; Thoracoscopy

\section{Introduction}

Surgery remains the gold standard treatment modality for esophageal and gastroesophageal junction (GEJ) cancer usually in combination with perioperative treatment. But operative procedure itself still remains a challenging task for surgeons. Curative surgical therapy for esophagogastric cancer has three fundamental principles: (1) resection of part or all of the affected organs (esophagus, stomach) with adequate clearance of the primary tumor, (2) removal of local and regional lymph nodes to eliminate potential metastatic disease, and (3) restoration of gastrointestinal continuity with satisfactory function. During the past century, a wide variety of different techniques in pursuit of these principles have been described, but to date no consensus has been reached as to which approach is ideal. Radical resections involving a thoracotomy and laparotomy in theory offer the greatest chance for long-term disease-free survival but are associated with substantial morbidity and significant impairment of postsurgical quality of life [1].

Less invasive techniques, although perhaps better tolerated, may fail to achieve the same oncologic goals. With the advancement of videoscopic technology and its application to surgery from the late 1990s onward, the concept of a minimally invasive resection using thoracoscopic and laparoscopic rather than open approaches has been an attractive aim. The intention is to achieve a curative resection but with substantially less surgical trauma relating to operative access. Initial enthusiasm was, however, dampened when early case series reported only limited success in achieving these aims [2,3]. Not until the experience described by Luketich et al. [4] did it become apparent that minimally invasive esophagectomy (MIE) might offer a viable alternative to traditional open surgery. Advanced Minimally invasive surgery for visceral organs has been a new evolving trend in Nepal as well, but to the best of our knowledge, MIE has been never been performed elsewhere in Nepal. The aim of our study is to assess the preliminary safety profile and early results of this approach in Nepalese context, with limited technical resources.

\section{Materials and Methods}

A prospective study of the patients with the diagnosis of upper esophageal, middle esophageal carcinoma, carcinoma of GEJ Siewert's type I and II was conducted during the period of mid 2010 till end of 2011 (one and half years). The study protocol was approved by the institute. Primary evaluation of the patients was done with routine blood tests, biochemistry profile, ECG, spirometry, esophagogastroscopy and $\mathrm{CT}$ chest and abdomen. Bronchoscopy was done for upper and middle esophageal lesions to exclude the tracheo-bronchial invasion.

*Corresponding author: Binay Thakur, Department of Surgical Oncology, BP Koirala Memorial Cancer Hospital, Bharatpur, Chitwan, Nepal, Tel: +977-56525725; E-mail: binaythakur@hotmail.com

Received March 17, 2012; Accepted April 28, 2012; Published April 30, 2012

Citation: Thakur B, Hui L, Devkota M, Xin C, Lama R (2012) Minimally Invasive Esophagectomy/Gastroesophagectomy for Cancer. Is it Safe in Nepalese Context? J Cancer Sci Ther 4: 102-105. doi:10.4172/1948-5956.1000120

Copyright: @ 2012 Thakur B, et al. This is an open-access article distributed unde the terms of the Creative Commons Attribution License, which permits unrestricted use, distribution, and reproduction in any medium, provided the original author and source are credited. 
Citation: Thakur B, Hui L, Devkota M, Xin C, Lama R (2012) Minimally Invasive Esophagectomy/Gastroesophagectomy for Cancer. Is it Safe in Nepalese Context? J Cancer Sci Ther 4: 102-105. doi:10.4172/1948-5956.1000120

\section{Inclusion and exclusion criteria}

Patients with ECOG 0-1 with non-cT4 and cM0 were included in the study. cT4 status was decided on the findings of CT films. In case of cT4 lesion, neoadjuvant treatment (chemoradiation or chemotherapy) was used and the response was assessed after its completion. The responders were subjected to the surgery. Patients with ECOG $>1$, $\mathrm{cM} 1$ disease and non-responders to the neoadjuvant treatment (if cT4 lesion) were excluded.

\section{Protocol}

Upper esophageal tumors were taken for definitive concurrent chemoradiation (Cisplatin-5-fluorouracil based chemotherapy along with radiation up to $50.4 \mathrm{~Gy}$ ). If there was residual lesion, the patients were subjected to surgery. Tumors of rest of the location were subjected primarily to surgery, if deemed resectable on the basis of CT films. The unresectable middle esophageal and GEJ type - I lesions were taken for neoadjuvant concurrent chemoradiation (cisplatin-5-FU chemotherapy with radiation therapy $45-50.4 \mathrm{~Gy}$ ) followed by surgery. Resectable GEJ type - II lesions were directly subjected to surgery. Final pathological staging was done in accordance with AJCC $7^{\text {th }}$ edition. R0 squamous cell subtype of tumors was simply followed up. T3 or T4 and any node positive adenocarcinoma of GEJ type - I and T2-4 or any node positive adenocarcinoma of GEJ - II were subjected to adjuvant chemoradiation (5-Fluorouracil - leucovorin with radiation therapy 45-50.4 Gy). The patients were given the right to choose either open approach or minimally invasive approach.

\section{Surgical technique}

Depending upon the location of the tumor, the following approaches were used:

\section{Totally MIE}

a. 3- incision: VATS - laparoscopy - neck

b. 2- incision: transhiatal laparoscopy - neck

c. 1-incision- laparoscopy

2. Hybrid

a. 3- incision - thoracotomy-laparoscopy-neck

b. 3-incision - VATS-laparotomy-neck

c. 2-incision-laparoscopy-thoracotomy

A single lung ventilation was done with the patient positioned in left lateral position. For VATS (video-assisted thoracoscopic surgery), three ports were used. An optical $10 \mathrm{~mm}$ port was introduced through the $8^{\text {th }}$ intercostal space along the mid axillary line. A $5 \mathrm{~mm}$ port for lung retraction was used in 4- 5 th intercostal space along anterior axillary line. A $3 \mathrm{~cm}$ incision for surgeon's working instruments was placed in $7^{\text {th }}-8^{\text {th }}$ intercostal space just posterior to the infrascapular line. Surgeon stood behind the patient. The infra-azygos dissection was performed through the $3 \mathrm{~cm}$ port whereas supraazygos dissection was performed through the later and the anterior $5 \mathrm{~mm}$ port. The azygos vein was either ligated and divided or preserved. As per the judgment of the surgical team, infra carinal nodal dissection or sometimes extended mediastinal nodal dissection to include nodes along left recurrent laryngeal nerve was performed.

Five laparoscopic ports were inserted. $10 \mathrm{~mm}$ optical port was introduced $3-4 \mathrm{~cm}$ above the umbilicus. Four $5 \mathrm{~mm}$ ports $(2$ in right and 2 in left sides of abdomen). Surgeon stood in the right side of the patient, camera operator in between the legs of the patient and the 1-st assistant in the left side of the patient. Gastro-colic ligament was opened; stomach was mobilized over right gastro-epiploic vessels. Left gastro-epipolic vessel, right gastric vessel and short gastric vessels were coagulated with bipolar forceps prior to division. The left gastric vessels were ligated twice with silk number 1 thread and divided. Upper nodal dissection (levels 1, 2, 7, 8, 9, 11, 10, 12 and 3, 4 as well for GEJ - II lesions) was performed. A $5 \mathrm{~cm}$ minilaparotomy including the optical port into the incision was performed and the specimen was retrieved. A gastric tube was constructed extracorporeally.

A 3-4 cm incision was made along left side of neck. Stomach was pulled up to the neck and gastroesophageal anastomosis was done in single layer (Gambee stitch).

\section{Statistical analysis}

Patients were analyzed for major and minor post operative complications, final staging and early survival result using SPSS 16.0.

\section{Results}

Thirty four patients underwent minimally invasive surgery for cancer of esophagus/ GEJ. Mean age of the patients was 57 years with $20(59 \%)$ males and 14 (41\%) females. 18 (53\%) and $11(32 \%)$ were smokers and alcoholic, respectively. 33 (97\%) presented with dysphagia, with mean duration of dysphagia of 3.3 months and mean weight loss of $8.2 \mathrm{~kg}$. Grade 0, II, III and IV dysphagia were present in $3 \%, 29 \%, 59 \%$ and $9 \%$, respectively. Basic parameters have been shown in Table 1.

Basic intra operative parameters and pathological findings have been shown in Table 2.

\begin{tabular}{|c|c|c|}
\hline Parameters & $\mathrm{N}$ & $\%$ \\
\hline $\begin{array}{l}\text { Location: } \\
\text { Upper esophagus } \\
\text { Mid esophagus } \\
\text { GEJ I } \\
\text { GEJ II }\end{array}$ & $\begin{array}{c}3 \\
13 \\
10 \\
8\end{array}$ & $\begin{array}{r}9 \\
38 \\
29 \\
24\end{array}$ \\
\hline $\begin{array}{l}\text { Treatment: } \\
\text { Preop CTRT }{ }^{\mathrm{a}}-\mathrm{S}^{\mathrm{b}} \\
\mathrm{S} \\
\mathrm{S}-\mathrm{CT}^{\mathrm{c}} \\
\mathrm{S}-\mathrm{CTRT}\end{array}$ & $\begin{array}{c}3 \\
28 \\
1 \\
2\end{array}$ & $\begin{array}{c}9 \\
82 \\
3 \\
6\end{array}$ \\
\hline $\begin{array}{l}\text { Approach: } \\
\text { Lap }^{d} \\
\text { Lap - thoracotomy } \\
\text { Lap THE } \\
\text { VATS }- \text { Lap - Neck } \\
\text { VATS - Laparotomy - neck } \\
\text { Lap - Thoracotomy - Neck }\end{array}$ & $\begin{array}{c}1 \\
1 \\
4 \\
4 \\
5 \\
19\end{array}$ & $\begin{array}{c}3 \\
3 \\
12 \\
12 \\
14 \\
56\end{array}$ \\
\hline $\begin{array}{l}\text { Level of anastomosis: } \\
\text { Chest } \\
\text { Neck }\end{array}$ & $\begin{array}{c}2 \\
32\end{array}$ & $\begin{array}{c}6 \\
94\end{array}$ \\
\hline $\begin{array}{l}\text { Nodal dissection: } \\
\text { Sampling } \\
\text { 2- FD } \\
\text { Extended } 2 \text { - FD } \\
\text { Total } 2 \text { - FD }\end{array}$ & $\begin{array}{c}4 \\
20 \\
2 \\
4\end{array}$ & $\begin{array}{c}13 \\
67 \\
7 \\
13\end{array}$ \\
\hline $\begin{array}{l}\text { Route of reconstruction: } \\
\text { Transmediastinal } \\
\text { Retrosternal }\end{array}$ & $\begin{array}{c}33 \\
1\end{array}$ & $\begin{array}{c}97 \\
3\end{array}$ \\
\hline
\end{tabular}

Table 1: Basic parameters. 
Mean ICU stay time was 2 days. Final pathological staging has been shown in Table 3 .

\section{Postoperative complication has been shown in Table 4 .}

The trend of surgery in our hospital has been shown in Figure 1. Because of short follow-up time, it was possible to calculate a six month survival, which is $97 \%$.

\section{Discussion}

Minimally invasive esophagectomy has not become widespread (unlike other minimally invasive procedures). It is still considered one of the most complex gastrointestinal surgical operations, and many questions remain unanswered as to the real advantages of applying minimally invasive techniques in this particular case. Mortality, morbidity, oncological radicality, and the cost of the procedure are just

\begin{tabular}{|l|c|c|}
\hline Parameters & N & $\%$ \\
\hline Mean operative time & $250 \mathrm{~min}$ & \\
\hline Mean Laparoscopy time & $145 \mathrm{~min}$ & \\
\hline Mean VATS time & $167 \mathrm{~min}$ & \\
\hline Mean tumor length & $7 \mathrm{~cm}$ & \\
\hline Mean dissected nodes & 22 & \\
\hline Mean positive nodes & 6 & \\
\hline R0 resection & 31 & 94 \\
\hline
\end{tabular}

Table 2: Intra-operative and pathological findings.

\begin{tabular}{|l|c|c|}
\hline pUICC $\left(7^{\text {th }}\right.$ edition $)$ & N & $\%$ \\
\hline Ib & 2 & 6 \\
Ila & 1 & 3 \\
IIb & 3 & 9 \\
IIIa & 6 & 18 \\
IIIb & 7 & 21 \\
IIIc & 13 & 40 \\
IV & 1 & 3 \\
\hline
\end{tabular}

Table 3: Final pathological stage.

\begin{tabular}{|l|c|c|}
\hline Parameters & N & $\%$ \\
\hline In-hospital death & 2 & 5.9 \\
\hline Transient hoarseness & 1 & 2.9 \\
\hline Cerebrovascular accident & 1 & 2.9 \\
\hline Anastomotic leak & 5 & 14.7 \\
\hline Hollow-viscous perforation & 1 & 2.9 \\
\hline Surgical site infection & 1 & 2.9 \\
\hline
\end{tabular}

Table 4: Postoperative complications.

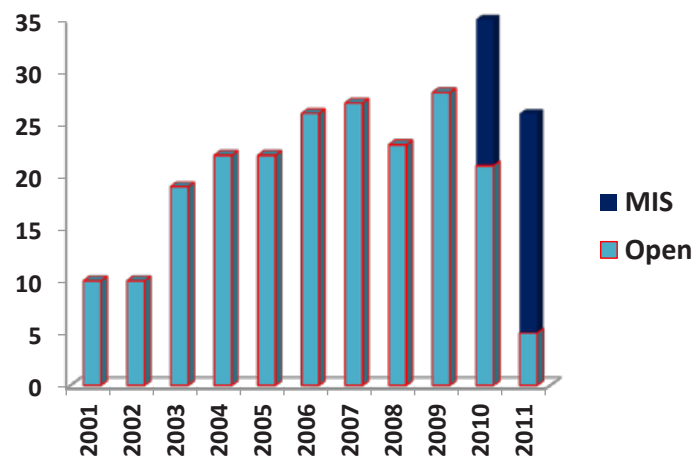

MIS: Minimally invasive surgery.

Figure 1: Trend of surgery. some of the topics under debate. Recent reviews focusing on the role of MIE have emphasized that the benefits of this approach are controversial due to the increased complexity involved [5]. Several comparative studies have been conducted between open esophagectomy (OE) and minimally invasive esophagectomy (MIE) [6,7]. Despite these studies, uncertainty about the advantages of one technique over the other persists. The question about the best approach for esophagectomy in esophageal cancer still remains unanswered.

Recently, the initial results of a phase II multiinstitutional study (Eastern Cooperative Oncology Group, ECOG 2202) to evaluate the results of MIE in a multiinstitutional setting were reported [4]. In this multicenter cooperative group trial, a total of 106 patients were enrolled. This study showed a low morbidity and mortality of less than $2 \%$. Median intensive care unit stay was 2 days. At a mean follow-up of 19 months, the estimated 3-year overall survival for the entire cohort was $50 \%$ ( $95 \%$ confidence interval, 35 to 65 ). Stage specific survival was similar to open series.

No randomized controlled trials currently exist for MIE, and it seems unlikely that any will be carried out given the known limitations of the procedure for locally advanced primary disease. This, in addition to the high dependence of MIE on advanced technical skill, has resulted in a slow acceptance of this technique as a valid or achievable standard of care. The available comparative data do suggest that MIE is as good as open oesophagectomy when performed by an appropriately skilled and experienced surgeon. Of particular note is the work of Luketich et al. $[4,8]$ who have reported the largest series of MIE (222 patients), albeit noncomparatively. Morbidity rates are at least comparable to open procedures in this series. Luketich's mortality rate of $1.3 \%$ is better than the mortality rate of $5.7 \%$ for transhiatal esophagectomy (THE) and 9.2\% for transthoracic esophagectomy (TTE) reported in the meta-analysis by Hulscher et al. [9] on open esophagectomy. It is unanimously accepted that total operative time is longer for MIE than for open surgery and except for the limitation caused by local oncological stage, this remains the major drawback in MIE. The best example of progression in MIE comes from Luketich et al. $[4,8]$, who have published serial data on their experience. The initial approach was with laparoscopic transhiatal esophagectomy, which changed to a thoracoscopic transhiatal technique over time. The initial description was of 77 patients who underwent MIE between August 1996 and September 1999 [8]. This has now been extended to include data for a total of 222 patients, the largest single series of any experience with MIE [4]. Median operative time for the first 20 cases was $450 \mathrm{~min}$, which decreased to $270 \mathrm{~min}$ thereafter. Anastomotic leak rate from initial experience was $9 \%$, which increased slightly to $11.7 \%$ over all 222 patients. Overall mortality rate for the 222 patients was $1.4 \%$. Progressively more advanced disease has become amenable to MIE through this series and should be taken into account when considering these data.

MIE can reduce the post-operative morbidity, in particular the respiratory complications which are most encountered. Different landmark studies have reported significantly low pulmonary complications rates using the minimally invasive transthoracic approach. Palanivelu et al. [10] report in their minimally invasive series of 130 patients in prone-position, $2.3 \%$ pulmonary complications whereas Luketich et al. [4] report in their series of 222 patients in left lateral decubitus MIE, $18 \%$ pulmonary complications. In contrast, Hulscher et al. [9] observed 57\% pulmonary complications in patients undergoing the traditional three-stage transthoracic open 
esophagectomy. Furthermore, median length of ICU stay was 1 day in the series of Palanivelu and Luketich whereas in the traditional series of Hulscher the ICU stay was 6 days. Oncologically, the type of resected specimen and lymph nodes are comparable with the open series and disease free and overall survival reported for MIE and traditional resection are quite comparable. These aforementioned landmark studies favor minimally invasive esophagectomy in terms of pulmonary complications and recovery.

A meta analysis was done by Nagpal et al. [11] comparing the minimally invasive esophagectomy (MIE) and hybrid MIE (HMIE) with open esophagectomy (OE). The authors included 12 studies with 672 patients in MIE and HMIE arm and 612 patients in OE arm $[6,7,12-21]$. The 30 day mortality were similar $(\mathrm{p}-\mathrm{ns})$. A trend towards lower anastomotic leak was found in MIE $(\mathrm{p}=0.08)$. Blood loss was significantly less in MIE group $(p<0.001)$. ICU stay $(p<0.001)$, total morbidity ( $\mathrm{p}=0.007)$, blood loss $(\mathrm{p}=0.03)$ were less in MIE group.

Our early results are comparable to the results obtained by other authors. A mortality rate of $5.9 \%$, mean ICU stay of 2 days and major post-operative complications are in acceptable range. The final pathological stage (79\% in pathological stage III) suggests the late arrival of the patients to the hospital, which in other terms may suggest that MIE is safe in locally advanced tumors as well. Our study mainly focused on the safety profile as this kind of surgery has never been reported earlier in Nepal. There were several drawbacks of our study, namely lack of use of endoscopic ultrasound (EUS) for preoperative $\mathrm{T}$ and $\mathrm{N}$ staging of the patients, only $12 \%$ of esophageal lesions ( 3 out of 26 patients) undergoing preoperative chemoradiation, none of GEJ tumors being considered for preoperative chemotherapy and a noncomparative study. Due to the lack of EUS at our center, it was not used. As approximately $70 \%$ patients had grade III-IV dyspagia with gross nutritional impairment and mostly poor economical status in order to afford for perioperative total pareneteral nutrition, the usual protocol at our hospital is to take the patient directly for surgery if deemed resectable on CT films. Our previous results in 100 patients with esophageal cancer undergoing open surgery with the similar protocol had shown final pathological stages III, IVa and IVb (UICC $6^{\text {th }}$ edition) in $46.6,4.9$ and $12.6 \%$ cases, respectively [22]. The overall 4 -year survival for the whole group was $20 \%$, which is in acceptable range. Besides, lack of vessel sealing devices and endo staplers prolonged the surgical time. Our trend for surgery for esophageal and GEJ cancer clearly shows a trend favoring MIE.

Till date, there has been no randomized controlled trial of open vs. MIE. Recently, a multicentric TIME - trial is being undertaken, comparing the traditional open approach with minimally invasive approach [23]. Thoracic resectable lesions and tumors of GEJ type I are eligible for inclusion. Cervical esophageal cancer and prior thoracic surgery are indications for exclusion. The results are awaited.

\section{Discussion}

Our preliminary results are encouraging and MIE may be safely recommended for Nepalese patients though a longer follow-up is needed to validate the survival results.

\section{References}

1. Griffin SM, Shaw IH, Dresner SM (2002) Early complica tions after Ivor Lewis subtotal esophagectomy with two-field lymphadenectomy: risk factors and management. J Am Coll Surg 194: 285-297.

2. Dexter SP, Martin IG, McMahon MJ (1996) Radical thoracoscopic esophagectomy for cancer. Surg Endosc 10: 147-151.
3. Peracchia A, Rosati R, Fumagalli U, Bona S, Chella B (1997) Thoracoscopic esophagectomy: are there benefits? Semin Surg Oncol 13: 259-262.

4. Luketich JD, Pennathur A, Catalano PJ, Swanson SJ, de Hoyos AL, et al. (2009) Results of a phase II multicenter study of minimally invasive esophagectomy (Eastern Cooperative Oncology Group Study E2202). J Clin Oncol 27: 15s.

5. Wu PC, Posner MC (2003) The role of surgery in the management of oesophageal cancer. Lancet Oncol 4: 481-488.

6. Smithers BM, Gotley DC, Martin I, Thomas JM (2007) Comparison of the outcomes between open and minimally invasive esophagectomy. Ann Surg 245: $232-240$

7. Shiraishi T, Kawahara K, Shirakusa T, Yamamoto S, Maekawa T (2006) Risk analysis in resection of thoracic esophageal cancer in the era of endoscopic surgery. Ann Thorac Surg 81: 1083-1089.

8. Luketich JD, Schauer PR, Christie NA, Weigel TL, Raja S, et al. (2000) Minimally invasive esophagectomy. Ann Thorac Surg 70: 906-911.

9. Hulscher JB, van Sandick JW, de Boer AG, Wijnhoven BP, Tijssen JG, et al (2002) Extended transthoracic resection compared with limited transhiata resection for adenocarcinoma of the esophagus. N Eng J Med 347: 1662-1669.

10. Palanivelu C, Prakash A, Senthilkumar R, Senthilnathan P, Parthasarathi R, et al. (2006) Minimally invasive esophagectomy: thoracoscopic mobilization of the esophagus and mediastinal lymphadenectomy in prone position--experience of 130 patients. J Am Coll Surg 203: 7-16.

11. Nagpal K, Ahmed K, Vats A, Yakoub D, James D, et al. (2010) Is minimally invasive surgery beneficial in the management of esophageal cancer? A metaanalysis. Surg Endosc 24: 1621-1629.

12. Bernabe KQ, Bolton JS, Richardson WS (2005) Laparoscopic hand-assisted versus open transhiatal esophagectomy: a case-control study. Surg Endosc 19: 334-337.

13. Benzoni E, Terrosu G, Bresadola V, Uzzau A, Intini S, et al. (2007) A comparative study of the transhiatal laparoscopic approach versus laparoscopic gastric mobilisation and right open transthoracic esophagectomy for esophageal cancer management. J Gastrointestin Liver Dis 16: 395-401.

14. Bonavina L, Bona D, Binyom PR, Peracchia A (2004) A laparoscopy-assisted surgical approach to esophageal carcinoma. J Surg Res 117: 52-57.

15. Braghetto I, Csendes A, Cardemil G, Burdiles P, Korn O, et al. (2006) Open transthoracic or transhiatal esophagectomy versus minimally invasive esophagectomy in terms of morbidity, mortality and survival. Surg Endosc 20 1681-1686.

16. Fabian T, Martin JT, McKelvey AA, Federico JA (2008) Minimally invasive esophagectomy: a teaching hospital's first year experience. Dis Esophagus 21 220-225.

17. Law S, Fok M, Chu KM, Wong J (1997) Thoracoscopic esophagectomy for esophageal cancer. Surgery 122: 8-14.

18. Martin DJ, Bessell JR, Chew A, Watson DI (2005) Thoracoscopic and laparoscopic esophagectomy: initial experience and outcomes. Surg Endosc 19: $1597-1601$

19. Nguyen NT, Follette DM, Wolfe BM, Schneider PD, Roberts P, et al. (2000) Comparison of minimally invasive esophagectomy with transthoracic and transhiatal esophagectomy. Arch Surg 135: 920-925.

20. Osugi H, Takemura M, Higashino M, Takada N, Lee S, et al. (2003) A comparison of video-assisted thoracoscopic oesophagectomy and radical lymph node dissection for squamous cell cancer of the oesophagus with open operation. Br J Surg 90: 108-113.

21. Van den Broek WT, Makay O, Berends FJ, Yuan JZ, Houdijk AP, et al. (2004) Laparoscopically assisted transhiatal resection for malignancies of the distal esophagus. Surg Endosc 18: 812-817.

22. Thakur B, Zhang CS, Meng XL, Bhaktaman S, Bhurtel S, et al. (2011) Eight Eight-year experience in esophageal cancer surgery. Indian J Cancer 48: 3439.

23. Biere SS, Maas KW, Bonavina L, Garcia JR, van Berge Henegouwen M, et al. (2011) Traditional invasive vs. minimally invasive esophagectomy: a multicenter, randomized trial (TIME-trial). BMC Surg 11: 2. 